\title{
LINKING SOURCE AND EFFECT: RE-SUSPENDED SOIL LEAD, AIR LEAD, AND CHILDREN'S BLOOD LEAD LEVELS IN DETROIT, MICHIGAN
}

Sammy Zahran, Mark A.S. Laidlaw, Shawn P. McElmurry, Gabriel M. Filippelli, Mark Taylor

\section{SUPPORTING INFORMATION}

Sample statistics (proportion) from Michigan Department of Community Health (MDCH)

dataset: male (0.51), female (0.49); age $<1(0.05)$, age $1(0.21)$, age $2(0.16)$, age $3(0.18)$, age 4 (0.19), age $5(0.09)$, age $6(0.05)$, age $7(0.02)$, age $\geq 8(0.04)$; venous draw $(0.78)$, capillary draw (0.22); January (0.07), February (0.07), March (0.08), April (0.08), May (0.08), June (0.07), July (0.08), August (0.13), September (0.12), October (0.09), November (0.07), December (0.05); year 2001 (0.10), 2002 (0.10), 2003 (0.11), 2004 (0.11), 2005 (0.11), 2006 (0.12), 2007 (0.12), 2008 (0.11), 2009 (0.11); and $\geq 5 \mu \mathrm{g} / \mathrm{dL}(0.33),<5 \mu \mathrm{g} / \mathrm{dL}(0.67)$.

Spatial and Temporal Resolution: Given that emissions drift spatially it was not clear a priori what the most appropriate unit is for capturing the point source risk of a $\mathrm{Pb}$ emitting facility. Data analysis using both measures $(1=\mathrm{Pb}$ facility in census tract of child residence; or $1=\mathrm{Pb}$ facility in zip code of child residence) were conducted before hand, and the zip code defined measure performed more consistently with stronger model fit statistics.

In Figure 1 we graph monitor data for both air $\mathrm{Pb}$ and air soil. The statistically optimal relationship is the daily time-step. To observe the association between air $\mathrm{Pb}$ and blood $\mathrm{Pb}$ temporally required alteration of the air $\mathrm{Pb}$ time step (to monthly) to match the time step of child blood $\mathrm{Pb}$. To protect the privacy of children, the Michigan Department of Community Health, Childhood Lead Prevention Poisoning Project only provided us with information on the month a 
child's blood was drawn. Further understanding of the temporal behavior of blood $\mathrm{Pb}$ concentrations may be evaluated in future studies if data with the appropriate resolution can be used. 
Table SI1. Fixed effect regression coefficients predicting the natural $\log$ of blood $\mathrm{Pb}$ in children as a function of month of blood draw, 2001-2009. Notes: standard errors in parentheses; $* * * \mathrm{p}<0.01, * * \mathrm{p}<0.05$; model controls for year of blood draw, gender, child age, and blood draw type (capillary $=1$, venous $=0$ ).

\begin{tabular}{|c|c|c|}
\hline & $\begin{array}{l}\text { Child BLL } \\
\text { Semi-elasticity }\end{array}$ & $95 \% C I$ \\
\hline February & $\begin{array}{l}-0.022 * * * \\
(0.006)\end{array}$ & {$[-.034$ to -.010$]$} \\
\hline March & $\begin{array}{l}-0.020^{* * * *} \\
(0.006)\end{array}$ & [-.032 to -.008$]$ \\
\hline April & $\begin{array}{l}-0.007 \\
(0.006)\end{array}$ & [-.018 to .005$]$ \\
\hline May & $\begin{array}{l}0.024 * * * \\
(0.006)\end{array}$ & [.013 to .036] \\
\hline June & $\begin{array}{l}0.079 * * * \\
(0.006)\end{array}$ & [.067 to .091] \\
\hline July & $\begin{array}{l}0.132 * * * \\
(0.006)\end{array}$ & [.121 to .144$]$ \\
\hline August & $\begin{array}{l}0.127^{* * * *} \\
(0.006)\end{array}$ & [.117 to .138$]$ \\
\hline September & $\begin{array}{l}0.122 * * * \\
(0.006)\end{array}$ & [.111 to .132$]$ \\
\hline October & $\begin{array}{l}0.065^{* * * *} \\
(0.006)\end{array}$ & {$[.054$ to .077$]$} \\
\hline November & $\begin{array}{l}0.017 * * * \\
(0.006)\end{array}$ & [.005 to .029] \\
\hline December & $\begin{array}{l}-0.0255^{* * *} \\
(0.007)\end{array}$ & {$[-.038$ to -.013$]$} \\
\hline Constant & $\begin{array}{l}1.029 * * * \\
(0.007)\end{array}$ & [1.015 to 1.043$]$ \\
\hline $\mathrm{N}$ & 362,646 & \\
\hline$\rho$ & 0.307 & \\
\hline$R^{2 \text { within }}$ & 0.116 & \\
\hline$R^{2}$ between & 0.139 & \\
\hline$R^{2 \text { overall }}$ & 0.108 & \\
\hline$F$ & 1976.8 & \\
\hline $\mathrm{N}_{\text {census tracts }}$ & 728 & \\
\hline
\end{tabular}


Traffic Induced Resuspension: Research indicates that a large fraction of road dust contains soil particles (approximately one-third according to Harrison, et al. [1]). Many trace metals observed in the IMPROVE database are found to be indicative of traffic induced resuspension. Specifically, iron, copper, antimony, barium, aluminum and zinc are associated with automobile wear [1-5]. Recent data presented by Harrison, Jones, Gietl, Yin and Green [1] demonstrated that the proportion of air particulate matter associated with brake dust and tire wear can be approximated based on barium and zinc concentrations. This association is consistent with the findings of others [5, 6]. Based on the result of Harrison, Jones, Gietl, Yin and Green [1], barium and zinc concentrations were multiplied by scaling factors of 91 and 50, respectively. The sum of these two products serves as a proxy for the influence of traffic induced resuspension.

While data on barium and zinc concentrations are not as comprehensive as for atmospheric $\mathrm{Pb}$ and soil, there are 409 monitor readings (over the time period of interest) where atmospheric $\mathrm{Pb}$ and (barium*91+zinc*50) are jointly observed. The results of the regression analysis that includes this traffic related proxy (Table SI2) suggest that resuspended soil is a more important source of atmospheric $\mathrm{Pb}$ than resuspended road dust. In Model 2 we observe that $1 \%$ increase in our road dust proxy is associated with a $0.255 \%$ increase in atmospheric Pb. In Model 3, results show that the elasticity of air $\mathrm{Pb}$ with respect to road dust decreases to $0.167 \%$ when adjusting for the effect of atmospheric soil. Also in Model 3 we observe that the standardized Beta of atmospheric soil $(0.286)>$ the standardized Beta $(0.146)$ for our road dust proxy. While it is difficult (technically) to fully discriminate these sources, given the known interchange between road dust and soil, the above results suggest that resuspended soil in Detroit is likely the more important source. Also, for the purpose of establishing a plausible pathway from soil dust 
$\rightarrow$ air $\mathrm{Pb} \rightarrow$ blood $\mathrm{Pb}$, it should be noted that our coefficient on atmospheric soil is unchanged with the inclusion of our road dust proxy (Model 1 elasticity $=0.482$ vs. Model 3 elasticity $=$ $0.483)$.

Table SI2. Fixed effect regression coefficients predicting the natural $\log$ of Air $\mathrm{Pb}$ as a function of atmospheric soil and road dust. Note: standard errors in parentheses; $* * * \mathrm{p}<0.01, * * \mathrm{p}<0.05$

\begin{tabular}{|lcccccc|}
\hline & $\begin{array}{c}\text { Air Lead } \\
(l n)\end{array}$ & $\begin{array}{c}\text { Air Lead } \\
(l n)\end{array}$ & $\begin{array}{c}\text { Air Lead } \\
(l n)\end{array}$ & $\begin{array}{c}\text { Air Lead } \\
(\ln )\end{array}$ & $\begin{array}{c}\text { Air Lead } \\
(l n)\end{array}$ & $\begin{array}{c}\text { Air Lead } \\
(\ln )\end{array}$ \\
& Model 1 & Model 1 & Model 2 & Model 2 & Model 3 & Model 3 \\
& & & & & & \\
& elastcity & beta & elastcity & beta & elastcity & beta \\
\hline Air Soil $(l n)$ & $0.482^{* * *}$ & 0.321 & & & $0.483^{* * *}$ & 0.290 \\
& $(0.050)$ & & & & $(0.080)$ & \\
Road dust & & & $0.255^{* * *}$ & 0.223 & $0.167^{* * *}$ & 0.146 \\
$(l n)$ & & & & & & \\
& & & $(0.055)$ & & $(0.055)$ & \\
Constant & $-5.038^{* * *}$ & & $-5.720^{* * *}$ & & $-5.133^{* * *}$ & \\
& $(0.064)$ & & $(0.073)$ & & $(0.120)$ & \\
$\mathrm{N}$ & & & & & \\
$R^{2}$ & 817 & & 409 & & 409 & \\
$F$ & 0.103 & & 0.050 & & 0.128 & \\
\hline
\end{tabular}


Table SI3. Least squares regression coefficients predicting the natural log of air lead and air soil. Note: standard errors in parentheses; $* * * \mathrm{p}<0.01, * * \mathrm{p}<0.05,{ }^{*} \mathrm{p}<0.1$

\begin{tabular}{|c|c|c|c|c|c|c|c|c|}
\hline & $\begin{array}{c}\text { Air Lead }(\ln ) \\
\text { Model } 1 \\
\text { elastcity }\end{array}$ & $\begin{array}{l}\text { Air Lead } \\
(\ln ) \\
\text { Model } 1 \\
\text { beta }\end{array}$ & $\begin{array}{l}\text { Air Lead } \\
\quad(\ln ) \\
\text { Model } 2 \\
\text { elastcity }\end{array}$ & $\begin{array}{l}\text { Air Lead } \\
\quad(\ln ) \\
\text { Model } 2 \\
\text { beta }\end{array}$ & $\begin{array}{l}\text { Air Lead } \\
\quad(\ln ) \\
\text { Model } 3 \\
\text { elastcity }\end{array}$ & $\begin{array}{l}\text { Air Lead } \\
(\ln ) \\
\text { Model } 3 \\
\text { beta }\end{array}$ & $\begin{array}{l}\text { Air Soil } \\
\quad(\ln ) \\
\text { Model } 4 \\
\text { elastcity }\end{array}$ & $\begin{array}{l}\text { Air Soil } \\
(\ln ) \\
\text { Model } 4 \\
\text { beta }\end{array}$ \\
\hline Humidity (ln) & - & - & $\begin{array}{c}0.208 \\
(0.183)\end{array}$ & 0.045 & $\begin{array}{c}0.0433 \\
(0.186)\end{array}$ & 0.009 & $\begin{array}{c}-0.580 * * * \\
(0.111)\end{array}$ & -0.172 \\
\hline Sea Level Pressure $(\ln )$ & - & - & $\begin{array}{c}12.076^{* *} \\
(5.523)\end{array}$ & 0.088 & $\begin{array}{c}19.30 * * * \\
(5.571)\end{array}$ & 0.141 & $\begin{array}{c}17.75 * * * \\
(3.11)\end{array}$ & 0.19 \\
\hline Temperature $(\ln )$ & - & - & $\begin{array}{c}0.197 * * \\
(0.087)\end{array}$ & 0.091 & $\begin{array}{c}0.395 * * * \\
(0.085)\end{array}$ & 0.182 & $\begin{array}{c}0.455 * * * \\
(0.048)\end{array}$ & 0.302 \\
\hline Visibility $(\ln )$ & - & - & $\begin{array}{c}-0.328 * * \\
(0.134)\end{array}$ & -0.096 & $\begin{array}{c}-0.422 * * * \\
(0.137)\end{array}$ & -0.123 & $\begin{array}{c}-0.339 * * * \\
(0.082)\end{array}$ & -0.133 \\
\hline Wind Speed $(\ln )$ & - & - & $\begin{array}{c}-0.152 * * \\
(0.072)\end{array}$ & -0.081 & $\begin{array}{c}-0.243 * * * \\
(0.073)\end{array}$ & -0.129 & $\begin{array}{c}-0.299 * * * \\
(0.042)\end{array}$ & -0.222 \\
\hline Air Soil $(\ln )$ & $\begin{array}{c}0.482 * * * \\
(0.050)\end{array}$ & 0.321 & $\begin{array}{c}0.388 * * * \\
(0.057)\end{array}$ & 0.259 & - & - & - & - \\
\hline Constant & $\begin{array}{c}-5.038 * * * \\
(0.064)\end{array}$ & & $\begin{array}{c}-46.874 * * * \\
(19.114)\end{array}$ & & $\begin{array}{c}-71.58 * * * \\
(19.29)\end{array}$ & & $\begin{array}{c}-59.62 * * * \\
(10.79)\end{array}$ & \\
\hline $\mathrm{N}$ & 817 & & 815 & & 815 & & 1,022 & \\
\hline$R^{2}$ & 0.103 & & 0.132 & & 0.082 & & 0.249 & \\
\hline$F$ & 93.63 & & 20.43 & & 14.41 & & 67.41 & \\
\hline
\end{tabular}


Table SI4. Least squares regression coefficients predicting weather-adjusted air lead and air soil. Note: standard errors in parentheses; ${ }^{* * *} \mathrm{p}<0.01,{ }^{* *} \mathrm{p}<0.05,{ }^{*} \mathrm{p}<0.1$.

\begin{tabular}{|c|c|c|c|c|}
\hline & $\begin{array}{c}\text { Air Lead } \\
(\ln ) \\
\text { Model } 1 \\
\text { Semi-elasticity }\end{array}$ & $\begin{array}{l}\text { Standardized Air } \\
\text { Lead }(\ln ) \\
\text { Model } 2 \\
b\end{array}$ & $\begin{array}{c}\text { Air Soil } \\
(\ln ) \\
\text { Model } 3 \\
\text { Semi-elasticity }\end{array}$ & $\begin{array}{c}\text { Standardized Air } \\
\text { Soil }(\ln ) \\
\text { Model } 4 \\
b\end{array}$ \\
\hline February & $\begin{array}{c}0.0185 \\
(0.0366)\end{array}$ & $\begin{array}{l}0.0693 \\
(0.137)\end{array}$ & $\begin{array}{c}0.0604 \\
(0.0412)\end{array}$ & $\begin{array}{c}0.184 \\
(0.126)\end{array}$ \\
\hline March & $\begin{array}{c}0.123 * * * \\
(0.0353)\end{array}$ & $\begin{array}{c}0.459 * * * \\
(0.132)\end{array}$ & $\begin{array}{c}0.217 * * * \\
(0.0397)\end{array}$ & $\begin{array}{c}0.664 * * * \\
(0.121)\end{array}$ \\
\hline April & $\begin{array}{c}0.188 * * * \\
(0.0357)\end{array}$ & $\begin{array}{c}0.702 * * * \\
(0.133)\end{array}$ & $\begin{array}{c}0.400 * * * \\
(0.0402)\end{array}$ & $\begin{array}{c}1.221 * * * \\
(0.123)\end{array}$ \\
\hline May & $\begin{array}{c}0.240 * * * \\
(0.0347)\end{array}$ & $\begin{array}{c}0.898 * * * \\
(0.130)\end{array}$ & $\begin{array}{c}0.439 * * * \\
(0.0391)\end{array}$ & $\begin{array}{c}1.340 * * * \\
(0.119)\end{array}$ \\
\hline June & $\begin{array}{c}0.352 * * * \\
(0.0353)\end{array}$ & $\begin{array}{c}1.315 * * * \\
(0.132)\end{array}$ & $\begin{array}{c}0.558 * * * \\
(0.0397)\end{array}$ & $\begin{array}{c}1.704 * * * \\
(0.121)\end{array}$ \\
\hline July & $\begin{array}{c}0.357 * * * \\
(0.0350)\end{array}$ & $\begin{array}{c}1.335 * * * \\
(0.131)\end{array}$ & $\begin{array}{c}0.578 * * * \\
(0.0394)\end{array}$ & $\begin{array}{c}1.765 * * * \\
(0.120)\end{array}$ \\
\hline August & $\begin{array}{c}0.448 * * * \\
(0.0349)\end{array}$ & $\begin{array}{c}1.677 * * * \\
(0.130)\end{array}$ & $\begin{array}{c}0.622 * * * \\
(0.0393)\end{array}$ & $\begin{array}{c}1.900 * * * \\
(0.120)\end{array}$ \\
\hline September & $\begin{array}{c}0.403 * * * \\
(0.0359)\end{array}$ & $\begin{array}{c}1.508 * * * \\
(0.134)\end{array}$ & $\begin{array}{c}0.540 * * * \\
(0.0404)\end{array}$ & $\begin{array}{c}1.648 * * * \\
(0.123)\end{array}$ \\
\hline October & $\begin{array}{c}0.258 * * * \\
(0.0353)\end{array}$ & $\begin{array}{c}0.965 * * * \\
(0.132)\end{array}$ & $\begin{array}{c}0.390 * * * \\
(0.0397)\end{array}$ & $\begin{array}{c}1.191 * * * \\
(0.121)\end{array}$ \\
\hline November & $\begin{array}{c}0.211 * * * \\
(0.0355)\end{array}$ & $\begin{array}{c}0.788 * * * \\
(0.133)\end{array}$ & $\begin{array}{c}0.289 * * * \\
(0.0399)\end{array}$ & $\begin{array}{c}0.884 * * * \\
(0.122)\end{array}$ \\
\hline December & $\begin{array}{c}0.0934 * * * \\
(0.0359)\end{array}$ & $\begin{array}{c}0.349 * * * \\
(0.134)\end{array}$ & $\begin{array}{l}0.101 * * \\
(0.0404)\end{array}$ & $\begin{array}{c}0.308 * * \\
(0.123)\end{array}$ \\
\hline Constant & $\begin{array}{c}-5.839 * * * \\
(0.0253)\end{array}$ & $\begin{array}{c}-0.857 * * * \\
(0.0946)\end{array}$ & $\begin{array}{c}-1.578^{* * *} \\
(0.0285)\end{array}$ & $\begin{array}{c}-1.091 * * * \\
(0.0870)\end{array}$ \\
\hline $\begin{array}{l}\mathrm{N} \\
R^{2} \\
F\end{array}$ & $\begin{array}{l}1,022 \\
0.274 \\
34.58\end{array}$ & & $\begin{array}{l}1,022 \\
0.387 \\
57.86\end{array}$ & \\
\hline
\end{tabular}


Table SI5. Fixed effect regression coefficients predicting the natural log of blood $\mathrm{Pb}$ in Detroit children, 2001-2009. Note: standard errors in parentheses; $* * * \mathrm{p}<0.01, * * \mathrm{p}<0.05$.

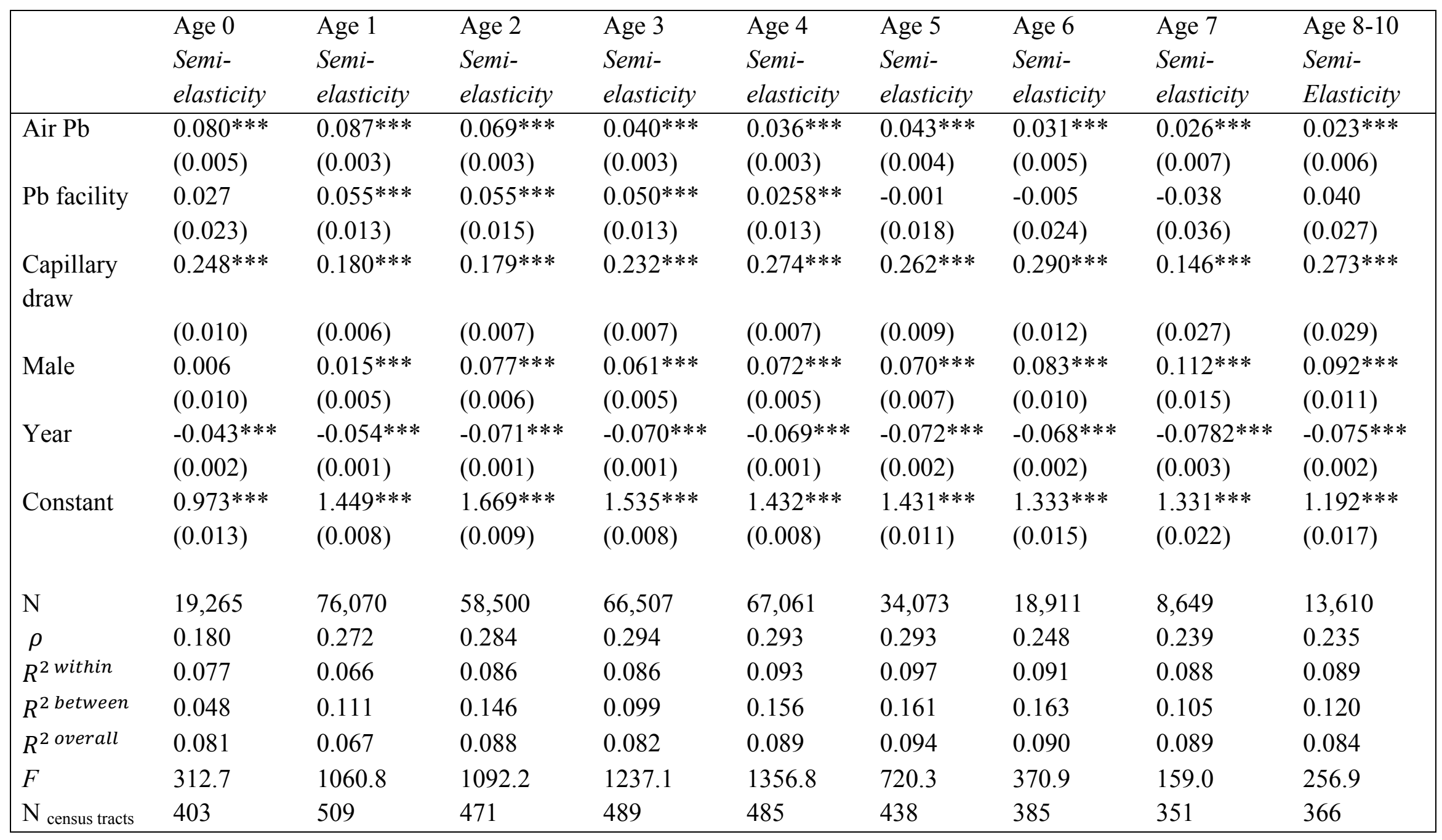


Table SI6. Fixed effect regression coefficients predicting the natural log of blood $\mathrm{Pb}$ in children as a function of soil residualized air $\mathrm{Pb}, 2001-2009$. Note: standard errors in parentheses; $* * * \mathrm{p}<0.01,{ }^{* *} \mathrm{p}<0.05$.

\begin{tabular}{|c|c|c|c|c|c|c|c|c|c|}
\hline & $\begin{array}{c}\text { Age } 0 \\
\text { Semi- } \\
\text { elasticity }\end{array}$ & $\begin{array}{c}\text { Age } 1 \\
\text { Semi- } \\
\text { elasticity }\end{array}$ & $\begin{array}{c}\text { Age } 2 \\
\text { Semi- } \\
\text { elasticity }\end{array}$ & $\begin{array}{c}\text { Age } 3 \\
\text { Semi- } \\
\text { elasticity }\end{array}$ & $\begin{array}{c}\text { Age } 4 \\
\text { Semi- } \\
\text { elasticity }\end{array}$ & $\begin{array}{c}\text { Age } 5 \\
\text { Semi- } \\
\text { elasticity }\end{array}$ & $\begin{array}{c}\text { Age } 6 \\
\text { Semi- } \\
\text { elasticity }\end{array}$ & $\begin{array}{l}\text { Age } 7 \\
\quad \text { Semi- } \\
\text { elasticity }\end{array}$ & $\begin{array}{c}\text { Age 8-10 } \\
\text { Semi- } \\
\text { Elasticity }\end{array}$ \\
\hline $\begin{array}{l}\text { Soil Residualized } \\
\text { Air } \mathrm{Pb}\end{array}$ & -0.018 & -0.008 & 0.008 & $-0.015 * *$ & -0.003 & -0.008 & 0.002 & 0.006 & -0.018 \\
\hline & $(0.014)$ & $(0.007)$ & $(0.008)$ & $(0.007)$ & $(0.010)$ & $(0.012)$ & $(0.020)$ & $(0.016)$ & $(0.014)$ \\
\hline $\mathrm{Pb}$ facility & $\begin{array}{c}0.033 \\
(0.023)\end{array}$ & $\begin{array}{c}0.056 * * * \\
(0.013)\end{array}$ & $\begin{array}{c}0.053 * * * \\
(0.015)\end{array}$ & $\begin{array}{c}0.047 * * * \\
(0.013)\end{array}$ & $\begin{array}{l}-0.000 \\
(0.018)\end{array}$ & $\begin{array}{l}-0.010 \\
(0.024)\end{array}$ & $\begin{array}{c}-0.041 \\
(0.036)\end{array}$ & $\begin{array}{c}0.040 \\
(0.027)\end{array}$ & $\begin{array}{c}0.033 \\
(0.023)\end{array}$ \\
\hline Capillary draw & $\begin{array}{c}0.255 * * * \\
(0.010)\end{array}$ & $\begin{array}{c}0.179 * * * \\
(0.006)\end{array}$ & $\begin{array}{c}0.176 * * * \\
(0.007)\end{array}$ & $\begin{array}{c}0.227 * * * \\
(0.007)\end{array}$ & $\begin{array}{c}0.251 * * * \\
(0.009)\end{array}$ & $\begin{array}{c}0.282 * * * \\
(0.012)\end{array}$ & $\begin{array}{c}0.142 * * * \\
(0.027)\end{array}$ & $\begin{array}{c}0.274 * * * \\
(0.029)\end{array}$ & $\begin{array}{c}0.255^{* * *} \\
(0.010)\end{array}$ \\
\hline Male & $\begin{array}{c}0.007 \\
(0.010)\end{array}$ & $\begin{array}{c}0.016 * * * \\
(0.005)\end{array}$ & $\begin{array}{c}0.077 * * * \\
(0.006)\end{array}$ & $\begin{array}{c}0.060 * * * \\
(0.005)\end{array}$ & $\begin{array}{c}0.067 * * * \\
(0.007)\end{array}$ & $\begin{array}{c}0.082 * * * \\
(0.011)\end{array}$ & $\begin{array}{c}0.111 * * * \\
(0.015)\end{array}$ & $\begin{array}{c}0.091 * * * \\
(0.011)\end{array}$ & $\begin{array}{c}0.007 \\
(0.010)\end{array}$ \\
\hline Year & $\begin{array}{c}-0.047 * * * \\
(0.002)\end{array}$ & $\begin{array}{c}-0.057 * * * \\
(0.001)\end{array}$ & $\begin{array}{c}-0.074 * * * \\
(0.001)\end{array}$ & $\begin{array}{c}-0.073 * * * \\
(0.001)\end{array}$ & $\begin{array}{c}-0.074 * * * \\
(0.002)\end{array}$ & $\begin{array}{c}-0.070 * * * \\
(0.002)\end{array}$ & $\begin{array}{c}-0.079 * * * \\
(0.003)\end{array}$ & $\begin{array}{c}-0.075 * * * \\
(0.003)\end{array}$ & $\begin{array}{c}-0.047 * * * \\
(0.002)\end{array}$ \\
\hline Constant & $\begin{array}{c}0.979 * * * \\
(0.014)\end{array}$ & $\begin{array}{c}1.454 * * * \\
(0.008)\end{array}$ & $\begin{array}{c}1.677 * * * \\
(0.009)\end{array}$ & $\begin{array}{c}1.553 * * * \\
(0.008)\end{array}$ & $\begin{array}{c}1.446^{* * *} \\
(0.011)\end{array}$ & $\begin{array}{c}1.343 * * * \\
(0.015)\end{array}$ & $\begin{array}{c}1.336^{* * *} \\
(0.022)\end{array}$ & $\begin{array}{c}1.195 * * * \\
(0.017)\end{array}$ & $\begin{array}{c}0.979 * * * \\
(0.014)\end{array}$ \\
\hline $\mathrm{N}$ & 19,265 & 76,070 & 58,500 & 66,507 & 67,061 & 34,073 & 18,911 & 8,649 & 13,610 \\
\hline$\rho$ & 0.172 & 0.268 & 0.286 & 0.294 & 0.293 & 0.290 & 0.246 & 0.241 & 0.236 \\
\hline$R^{2 \text { within }}$ & 0.063 & 0.052 & 0.077 & 0.083 & 0.090 & 0.093 & 0.089 & 0.086 & 0.087 \\
\hline$R^{2 \text { between }}$ & 0.082 & 0.131 & 0.128 & 0.089 & 0.152 & 0.175 & 0.172 & 0.096 & 0.116 \\
\hline$R^{2 \text { overall }}$ & 0.070 & 0.055 & 0.081 & 0.080 & 0.087 & 0.091 & 0.089 & 0.088 & 0.084 \\
\hline$F$ & 254.2 & 822.1 & 974.2 & 1190.2 & 1314.4 & 690.1 & 361.8 & 156.3 & 253.6 \\
\hline $\mathrm{N}_{\text {census tracts }}$ & 403 & 509 & 471 & 489 & 485 & 438 & 385 & 351 & 366 \\
\hline
\end{tabular}


Figure SI1. Scatterplot with median splines of adjusted child BLL (Monthly). Child BLL estimates (from Table SI1) are graphed on the monthly time-step, fitting the distribution with a median spline. Date of blood draw is on the x-axis, and predicted child BLL is on the y-axis.

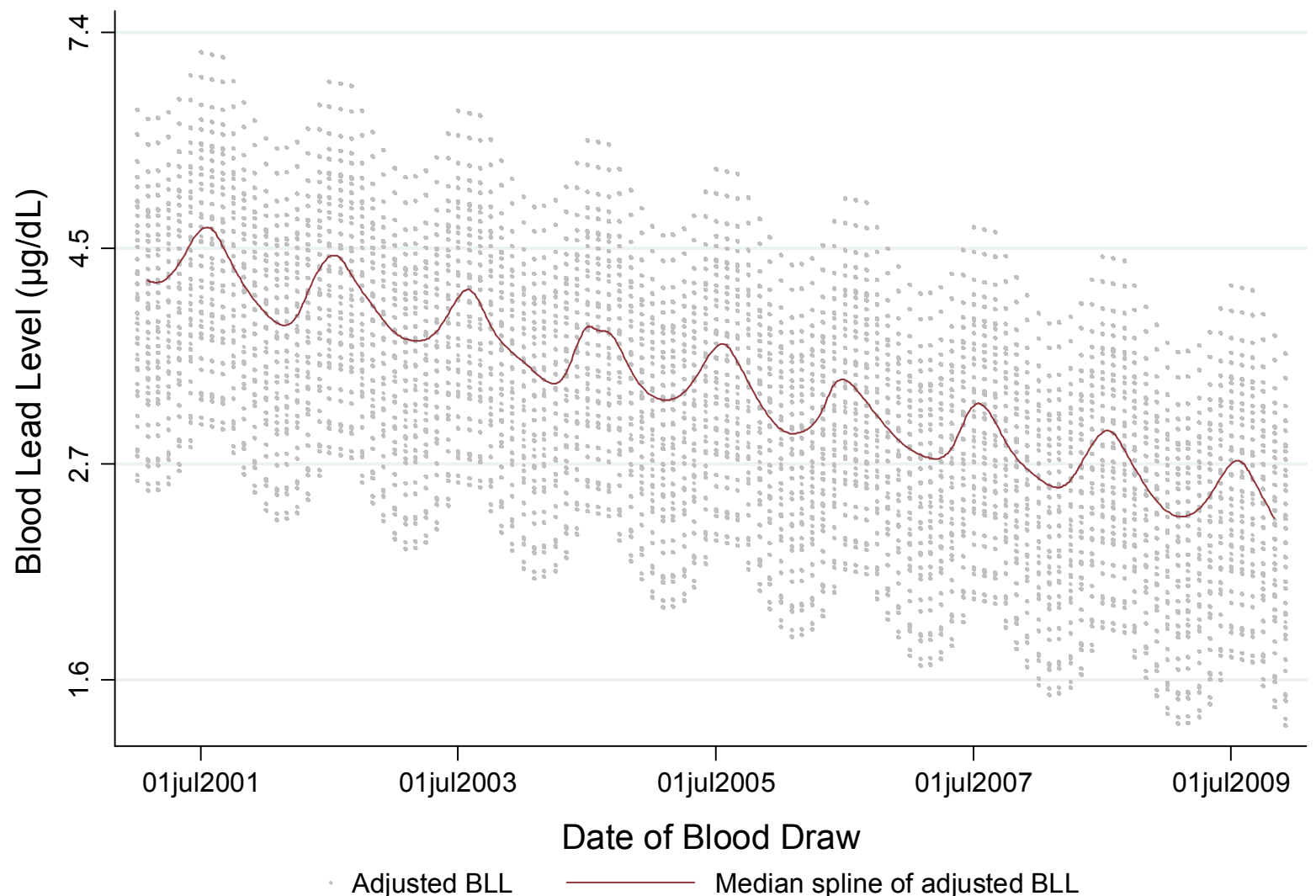


Spatial Variability of Atmospheric Pb Data: Air Pb data from Station 261630001 was presented because data from this station was most comprehensive over the observation period of child blood $\mathrm{Pb}$. To rule out the possibility that the temporal behavior of atmospheric $\mathrm{Pb}$ data from the station we used (station 261630001) is not a statistical aberration or whether our intuition/theoretical claims are robust to other IMPROVE stations, we evaluated air $\mathrm{Pb}$ data from all 4 stations located within the Detroit metropolitan area (site codes: 261630001; 261250010; 261630033; and 261630015). While atmospheric $\mathrm{Pb}$ values deviate from station to station in given instances, all station data show strikingly similar seasonal properties and all data match the observed seasonality in child blood $\mathrm{Pb}$ (see Figure SI2 below). Based on these results we are scientifically confident that the seasonal aspect of our hypothesized pathway is not an artifact of relying on data from the single station used (\#261630001). As our graphic shows, the level of $\mathrm{Pb}$-in-air (for any given instance) will vary spatially (or from one station to the next), but all station data fluctuate similarly in time. In addition to this analysis, it should be noted that we do account for unobserved heterogeneity with census tract fixed effects. Whatever operates at the scale of the census tract (and specific to each tract) that is unobserved (including nonrandom spatial variation in air $\mathrm{Pb}$ ) is corrected for in the estimated impact of air $\mathrm{Pb}$ on blood $\mathrm{Pb}$. $\mathrm{As} \mathrm{a}$ result the air $\mathrm{Pb}$ effect reported is unbiased by spatial variation. 
Figure SI2. Weather-adjusted air $\mathrm{Pb}$ data from all 4 stations located within the Detroit metropolitan area (site codes: 261630001; 261250010; 261630033; and 261630015)

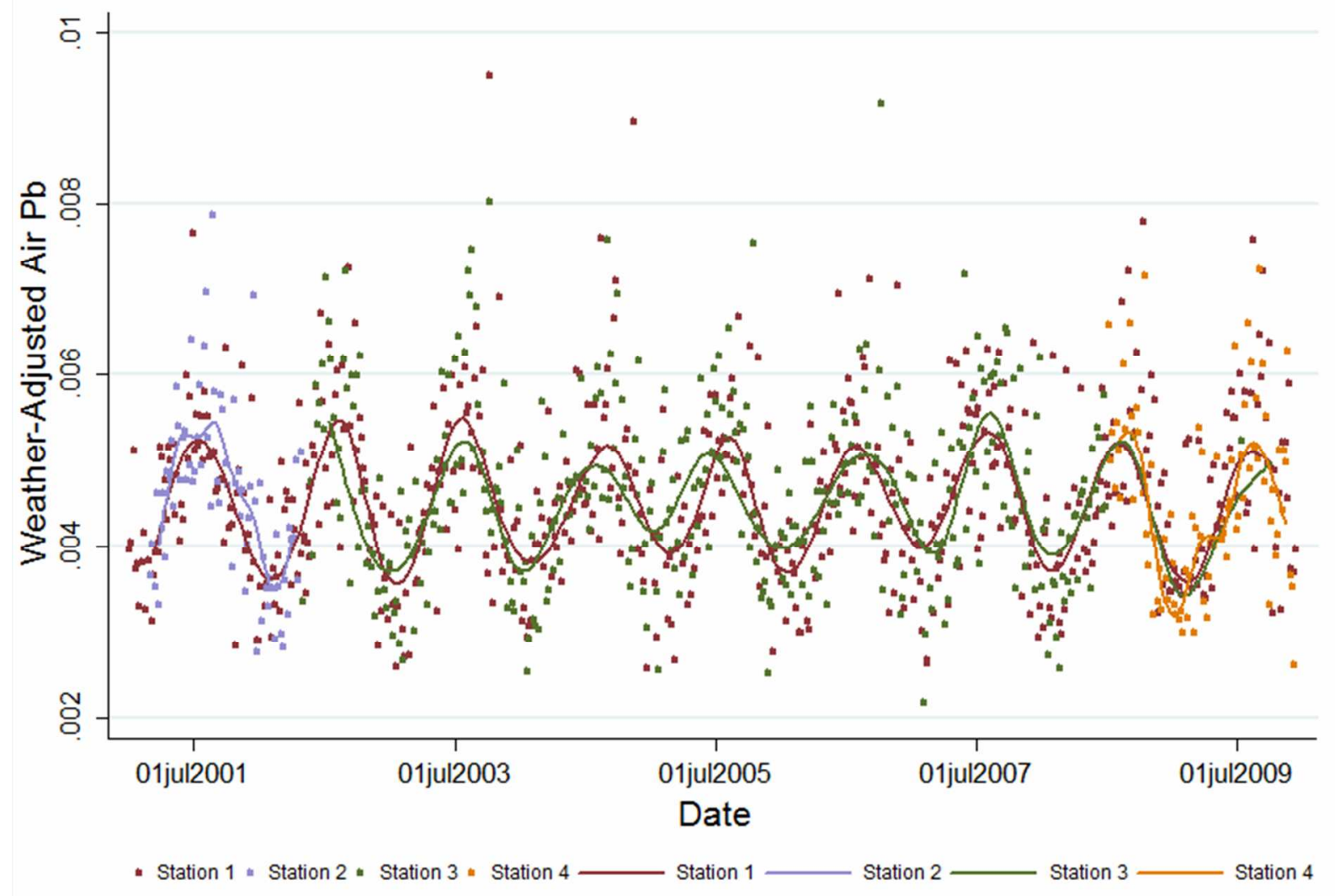


Effect of Weather Conditions: The resuspension patterns of soil and road dust are likely to vary significantly under different wind and weather conditions. For example, soil decreases significantly (to near zero) when soil is wet, the ground is frozen, or the ground is covered with snow. However, due to traffic induced resuspension, road dust is not likely to follow this pattern as strictly. To investigate possible differences in the contribution of $\mathrm{Pb}$ from soil versus road dust the Figure SI3 was developed. On the y-axis we plot standardized atmospheric concentration, where 0 equals the average. On the $\mathrm{x}$-axis, we divide station data into days with and without precipitation.

As observed in Table SI2, regression analyses show that atmospheric soil is a more important predictor of atmospheric $\mathrm{Pb}$ (as compared to atmospheric road dust). One plausible reason that soil outperforms road dust as the likely major source of atmospheric $\mathrm{Pb}$ is that road coverage in Detroit is a tiny fraction of soil coverage. In other cities, like New York, where the fraction of paved surfaces is greater, road dust may predominate. Theoretically, it may be the case that road dust may be more important than soil on an equal density basis. However, empirically, in the case of Detroit, given regression analyses describe above, the opposite seems more likely. 
Figure SI3. Standardized atmospheric concentration of $\mathrm{Pb}$, soil and road dust relative to days with and without precipitation

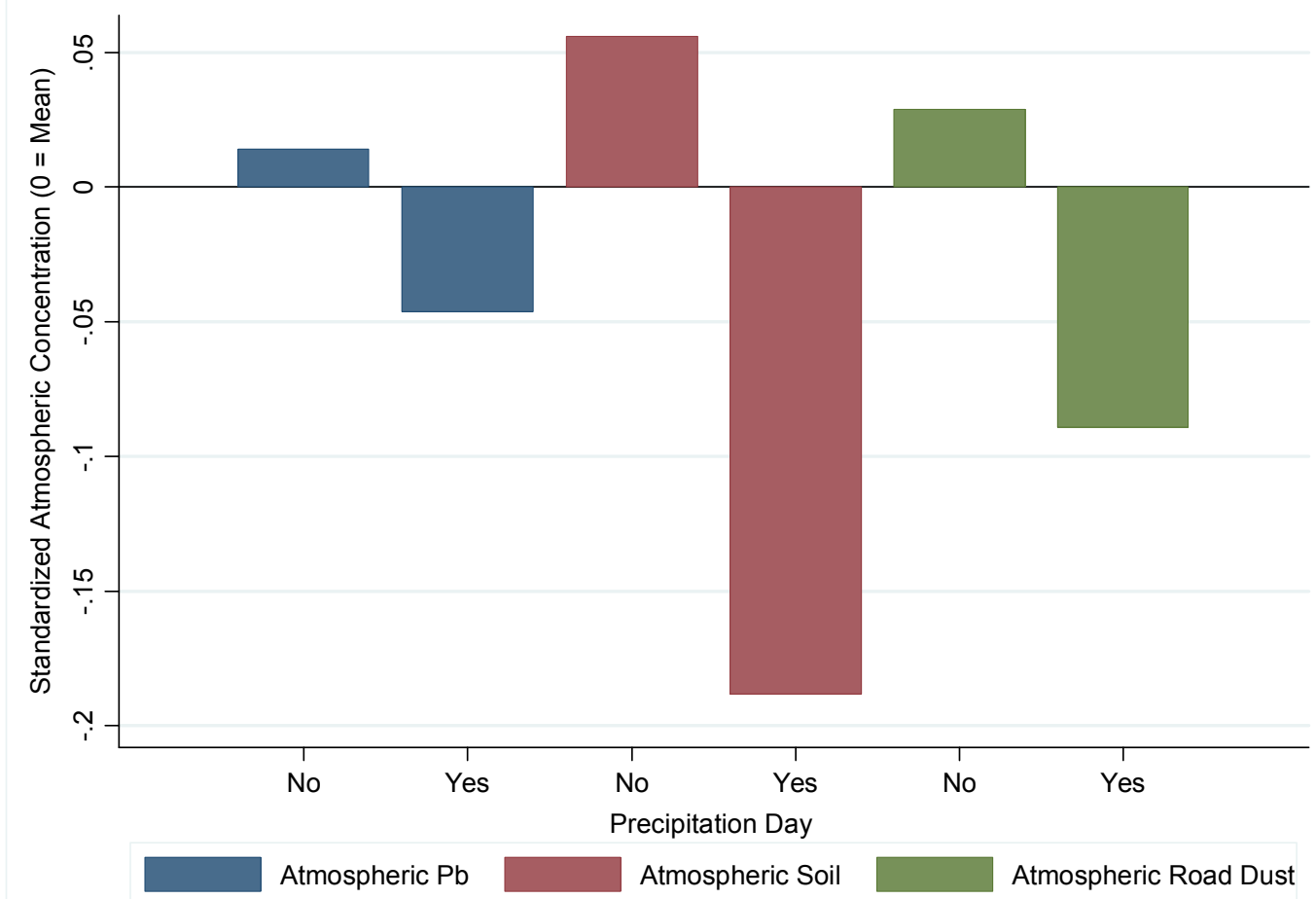




\section{ADDITIONAL REFERENCES:}

1. Harrison, R. M.; Jones, A. M.; Gietl, J.; Yin, J.; Green, D. C., Estimation of the Contributions of Brake Dust, Tire Wear, and Resuspension to Nonexhaust Traffic Particles Derived from Atmospheric Measurements. Environmental Science \& Technology 2012, 46, (12), 6523-6529.

2. Adachi, K.; Tainosho, Y., Characterization of heavy metal particles embedded in tire dust. Environ. Int. 2004, 30, (8), 1009-1017.

3. Amato, F.; Pandolfi, M.; Viana, M.; Querol, X.; Alastuey, A.; Moreno, T., Spatial and chemical patterns of PM10 in road dust deposited in urban environment. Atmos. Environ. 2009, 43, (9), 1650-1659.

4. Sternbeck, J.; Sjodin, A.; Andreasson, K., Metal emissions from road traffic and the influence of resuspension - results from two tunnel studies. Atmos. Environ. 2002, 36, (30), 4735-4744.

5. Weckwerth, G., Verification of traffic emitted aerosol components in the ambient air of Cologne (Germany). Atmos. Environ. 2001, 35, (32), 5525-5536.

6. Gietl, J. K.; Lawrence, R.; Thorpe, A. J.; Harrison, R. M., Identification of brake wear particles and derivation of a quantitative tracer for brake dust at a major road. Atmos. Environ. 2010, 44, (2), 141-146. 